\title{
Genome-wide association study of degenerative mitral valve disease in Maltese dogs
}

\author{
Chang-Min Lee ${ }^{1}$, Doo-Won Song ${ }^{2}$, Woong-Bin Ro ${ }^{2}$, Min-Hee Kang ${ }^{2}$, Hee-Myung Park ${ }^{2, *}$ \\ ${ }^{I}$ Department of Veterinary Laboratory Medicine, College of Veterinary Medicine, Chonnam National University, Gwangju 61186, Korea \\ ${ }^{2}$ Department of Veterinary Internal Medicine, College of Veterinary Medicine, Konkuk University, Seoul 05030, Korea
}

\begin{abstract}
Genome-wide association study (GWAS) is a powerful tool for identifying the genetic causes of various diseases. This study was conducted to identify genomic variation in Maltese dog genomes associated with degenerative mitral valve disease (DMVD) development and to evaluate the association of each biological condition with DMVD in Maltese dogs. DNA was extracted from blood samples obtained from 48 Maltese dogs (32 with DMVD and 16 controls). Genome-wide single nucleotide polymorphism (SNP) genotyping was performed. The top 30 SNPs from each association of various conditions and genetic variations were mapped to their gene locations. A total of 173,662 loci were successfully genotyped, with an overall genotype completion rate of $99.41 \%$. Quality control analysis excluded 46,610 of these SNPs. Manhattan plots were produced using allelic tests with various candidate clinical conditions. A significant peak of association was observed between mitral valve prolapse (MVP) and SNPs on chromosome 17. The present study revealed significant SNPs in several genes associated with cardiac function, including PDZ2, Armadillo repeat protein detected in velo-cardio-facial syndrome, catenin (cadherin-associated protein) alpha 3, low-density lipoprotein receptor class A domain containing protein 4, and sterile alpha motif domain containing protein 3 . To our knowledge, this is the first study of a genetic predisposition to DMVD in Maltese dogs. Although only a limited number of cases were analyzed, these data could be the basis for further research on the genetic predisposition to MVP and DMVD in Maltese dogs.
\end{abstract}

Keywords: Dogs; Genome-wide association study; Mitral valve; Mitral valve prolapse

\section{Introduction}

In genetic epidemiology, genome-wide association study (GWAS) is a relatively new technology for the identification of causative common genetic variants for important traits in livestock. GWAS mainly identifies the associations between single nucleotide polymorphisms (SNPs) and traits of interest in the whole genome, together with the phenotype [28]. Each sample of DNA from numerous genetic variants is described by using SNP genotyping, and if one of the variants is more frequently identified in the case group than the control group, then the SNP is considered associated with the trait [21].

GWAS is emerging as a favored method for understanding genetic and phenotypic variation. However, the potential usefulness of GWAS depends on the level of linkage disequilibrium (LD), since only a limited number of markers can be genotyped [32]. For analyzing genetic association in canines, the characteristic LD in the canine genome needs to be elucidated. According to a previous study, the $\mathrm{LD}$ in dogs is 20 to 50 times more extensive than that in humans, with more than 500,000 SNPs needing to be genotyped for whole-genome association studies in humans, but only approximately 10,000 SNPs required for comparative dog studies [25]. Consequently, given that dog breeds express specific phenotypic traits and incidences of genetic disease, GWAS for determination of LD could allow for the discovery of genes influencing breed-specific characteristics [25].

Myxomatous degeneration and secondary inappropriate internal environmental adaption of the mitral valve is a major cause of morbidity and mortality in dogs, with an incidence rate increasing with age in all dog breeds [5]. Degenerative mitral valve disease (DMVD) is the most common heart disease in dogs, accounting for almost $75 \%$ of all dogs with cardiac disorders [20]. DMVD is usually diagnosed in small breeds, with large breeds less prone to acquiring this disease [6]. DMVD is age-dependent, with the incidence of occurrence 
increasing in dogs over seven years of age, and, compared to females, males are more susceptible to DMVD [2].

Although GWAS could be a powerful tool for identifying the genetic causes of mitral valve diseases in humans, it would require a large number of cases and controls [20]. Controlled breeding has resulted in individual dog breeds that are genetically much more homogeneous than human populations [19]. In addition, LD in single breeds is large, which permits whole-genome association analysis with a smaller set of polymorphic markers [11]. This increases the ability to detect associations between phenotypes and genotypes confidently and helps to identify major factors in the molecular pathways underlying disease development [17].

The purpose of the present study was to identify genomic variations in the dog genome that affect DMVD development and to investigate the association between genetic polymorphisms and DMVD conditions.

\section{Materials and Methods}

\section{Animals}

Forty-eight Maltese dogs ( 32 with DMVD and 16 controls) were included in the present study. All Maltese dogs were randomly selected from dogs referred to the Veterinary Medical Teaching Hospital of Konkuk University between January 2014 and November 2015. The medical records, physical examinations, and echocardiography findings of all Maltese dogs were evaluated. Echocardiography was used to confirm the presence of mitral regurgitation and to evaluate structural abnormalities including mitral valve prolapse (MVP). Dogs with echocardiographic abnormalities suggestive of congenital heart disease or cardiomyopathic diseases were excluded. The criteria for inclusion as a control in the present study included Maltese dogs with a minimum age of 10 years, normal results of cardiovascular examinations such as echocardiography, and no pathologic conditions. The experimental design of the present study was approved by the University of Konkuk Institutional Animal Care and Use Committee (approval No. KU15115).

\section{American College of Veterinary Internal Medicine (ACVIM) class}

Maltese dogs diagnosed with DMVD were classified into three groups according to the ACVIM Consensus Statement, described previously [1], in which Stage B included dogs with DMVD that had never developed clinical signs associated with heart failure, Stage C included those with past or current clinical signs of heart failure, and Stage D included those with end-stage disease and clinical signs of heart failure that were refractory to standard treatment. The ACVIM Stage A group was excluded, as this group includes dogs having genetic factors such as the Cavalier King Charles Spaniel (CKCS) breed.

\section{Mitral valve prolapse}

In echocardiography, mitral regurgitation in dogs is associated with MVP, a systolic displacement of one or both leaflets to the atrial side of the plane of the mitral annulus [33]. The degree of MVP can be classified as mild, moderate, or severe [34]. In the present study, MVP was described as a leaflet prolapsing over the line from the middle of the elliptical echogenic area.

\section{Hematologic assessment}

Complete blood counts were performed using an automated system to evaluate the general condition of the dogs in the present study. The presence or absence of abnormalities was recorded. Leukocytosis is a condition characterized by a white blood cell count higher than $16.7 \times 10^{3} / \mu \mathrm{L}$ and thrombocytosis is characterized by a platelet count higher than $500 \times 10^{3} / \mu \mathrm{L}$.

\section{Vertebral heart scale}

In all radiographs, vertical and horizontal axes of the heart were measured and then converted to vertebral units in accordance with a method previously described [7]. The vertebral heart scale (VHS) was established as the sum of the short and long axes [18].

According to previous studies, enlargement of cardiac size groups has been divided using the VHS. The normal size group includes dogs under 10.7 on the VHS and the moderate group includes those having VHS values between 10.7 and 12.0. The severe group included dogs having VHS values greater than 12.0.

\section{Blood sample collection and DNA preparation}

One to two milliliters of blood were collected from the jugular vein of each dog for genomic DNA extraction. Before the collection of blood samples, informed owner consent and ethical committee approval were obtained. A 1-mL aliquot of the sample was added to an ethylenediaminetetraacetic acid (EDTA) anticoagulant tube, and the tubes were maintained on ice during transport and stored at $-80^{\circ} \mathrm{C}$ until use.

Genomic DNA was extracted from EDTA-treated whole blood using a commercial kit (QIAamp DNA Blood Mini Kit; Qiagen, Germany) according to the manufacturer's instructions. DNA concentrations were measured using a spectrometer. The A260/A280 ratio of all extracted samples was within the normal range of 1.7 to 1.9. The extracted DNA was stored at $-20^{\circ} \mathrm{C}$ until the following procedures were carried out.

\section{Genome-wide SNP genotyping and association study}

SNP genotyping was performed at the commercial laboratory using a BeadChip system (CanineHD BeadChip; Illumina, USA) containing 170,000 SNPs markers and an average of 70 SNP markers per megabase (Mb). DNA from 32 DMVD-affected dogs and 16 control dogs were analyzed. These samples were processed using a reading system (InfiniumR HD Assay Ultra 
system; Illumina) over three days. The DNA samples were prepared and quantitated according to the manufacturer's recommendations. The samples were then amplified and incubated for $20 \mathrm{~h}$. The next day, the amplified DNA was fragmented by applying restriction enzymes, and the fragmented DNA was then precipitated and resuspended. The prepared DNA samples were pipetted onto BeadChips and incubated at $48^{\circ} \mathrm{C}$ for $20 \mathrm{~h}$ for hybridization. On the third day, the hybridized samples were extended and stained using the BeadChips. The stained samples were visualized and scanned using a commercial platform (Illumina iScan platform; Illumina).

\section{Quality control and association analysis}

The iScan output was analyzed using specialized software (GenomeStudio software; Illumina), and the samples from both the DMVD-affected and control groups were combined to optimize genotype clustering. Polymorphic genotype data for each group were analyzed separately to examine levels of heterozygosity and deviation from the Hardy-Weinberg equilibrium.

A total of 170,000 SNPs displayed polymorphisms within the combined sample set, with greater than $99 \%$ of the SNPs included in acceptable genotype clusters. We excluded SNPs with genotyping call rates below $98 \%$ and those that were monomorphic or had minor allele frequencies less than $5 \%$ in our samples. SNPs in the X chromosome were excluded because of allelic imbalance. As the dogs were divided into DMVD-affected and control groups, a multidimensional scaling plot was prepared to assess for the presence of a tendentious sample distribution. A quantile-quantile (Q-Q) plot analysis was also performed to confirm whether the distribution of the inflation-corrected $p$ values deviated from the expected distribution under the null hypothesis of no genetic association. A genome inflation factor $(\lambda)$ was calculated by dividing the median value of the observed $p$ value by 0.456 to assess the potential population stratification of the present study [14].

Six different association analyses were conducted using 127,052 SNPs in a specialized program (Variation Suite v8; Golden Helix, USA) with or without adjustment for covariates. In addition, statistical analysis was conducted on these SNPs

Table 1. Characteristics of dogs in the present study

\begin{tabular}{lrrr}
\hline \multicolumn{1}{c}{ Variations } & \multicolumn{1}{c}{ DMVD } & \multicolumn{1}{c}{ Control } & \multicolumn{1}{c}{ Total } \\
\hline Total, n (\%) & $32(66.7)$ & $16(33.3)$ & $48(100.0)$ \\
Age, mean \pm SD (yr) & $10.4 \pm 1.7$ & $13.5 \pm 1.4$ & $11.4 \pm 2.2$ \\
Male (neutered), \% & $53.1(46.9)$ & $50.0(37.5)$ & $52.1(43.8)$ \\
Female (neutered), \% & $46.9(37.5)$ & $50.0(31.3)$ & $47.9(35.4)$ \\
\hline
\end{tabular}

DMVD, degenerative mitral valve disease. using the same program. A nominal $p<0.05$ was considered statistically significant. Proportions of samples with DMVD, MVP, leukocytosis, and thrombocytosis were compared using Fisher's exact tests. Multivariate linear regressions were performed to model the relationships between groups, including ACVIM classes and VHS groups.

\section{Identification of SNP genes and markers}

To identify the genes corresponding to the top 30 independent SNP associations in each analysis, SNP locus data were obtained from the University of California, Santa Cruz (UCSC) Genome Browser (Genome Bioinformatics Group, UCSC, USA). The gene annotations from the UCSC database were also used to predict the functional effects of variants on transcripts. The results show variant locations, including the coding sequence or intron of alternatively spliced gene transcripts.

\section{Results}

\section{Case selection and association condition group}

The present study included 25 males (21 castrated and four intact) and 23 females (17 spayed and six intact) with an average age of 11.6 years. The basic characteristics of the Maltese dogs are summarized in Table 1. Six different condition groups for the association study were divided by clinical manifestations of DMVD (Table 2).

Table 2. Number of dogs in each association analysis group divided by the clinical manifestation of DMVD

\begin{tabular}{clc}
\hline \multicolumn{1}{c}{ Condition } & Variable & No. of dogs \\
\hline \multirow{2}{*}{ DMVD } & Control & 16 \\
\multirow{3}{*}{ ACVIM class } & Phenotype & 32 \\
& Control & 16 \\
& Stage B & 10 \\
& Stage C & 17 \\
MVP & Stage D & 5 \\
\multirow{3}{*}{ Leuko-cytosis } & Control & 21 \\
\multirow{3}{*}{ Thrombo-cytosis } & Phenotype & 27 \\
& Negative & 15 \\
VHS grade & Positive & 9 \\
& Negative & 18 \\
& Positive & 7 \\
& Normal & 15 \\
& Moderate & 18 \\
\hline
\end{tabular}

DMVD, degenerative mitral valve disease; ACVIM, American College of Veterinary Internal Medicine; MVP, mitral valve prolapse; VHS, vertebral heart scale. 


\section{Multidimensional scaling test for stratification}

Stratification analysis by multidimensional scaling revealed that all but two dogs were within a single cluster (Fig. 1). Although two outliers were detected, the remaining dogs were closely clustered in the analysis $(<0.5 \%)$. Those two outliers were excluded in this association analysis because the two dogs

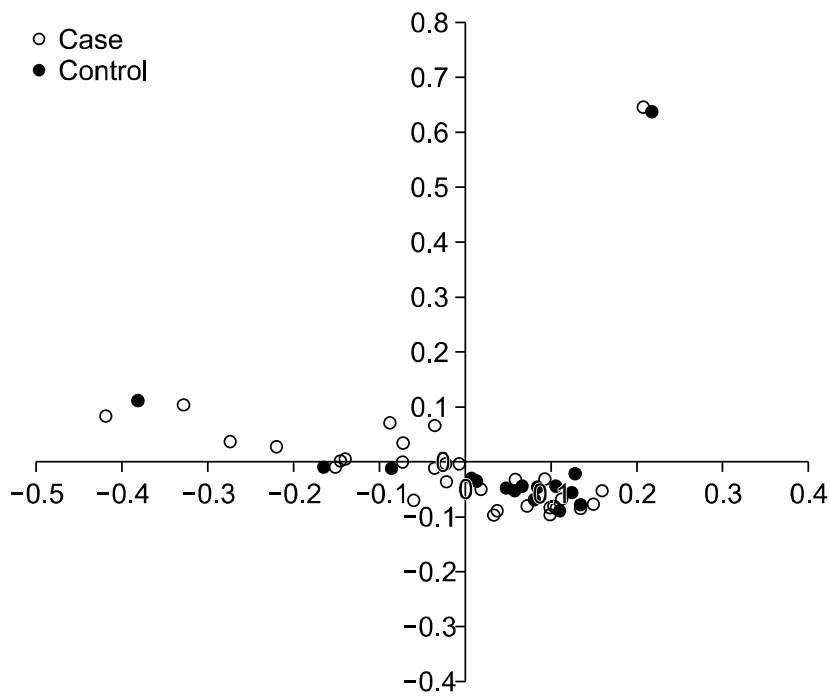

Fig. 1. Multidimensional scaling plot showing the distribution of case and control dogs in the present study. Those two outliers were excluded in this study because they were less likely to be purebred. were less likely to be purebred Maltese. Excluding the outliers, the results indicate that there was no population stratification within the study sample.

\section{Genotyping result}

A total of 173,662 loci were successfully genotyped in the

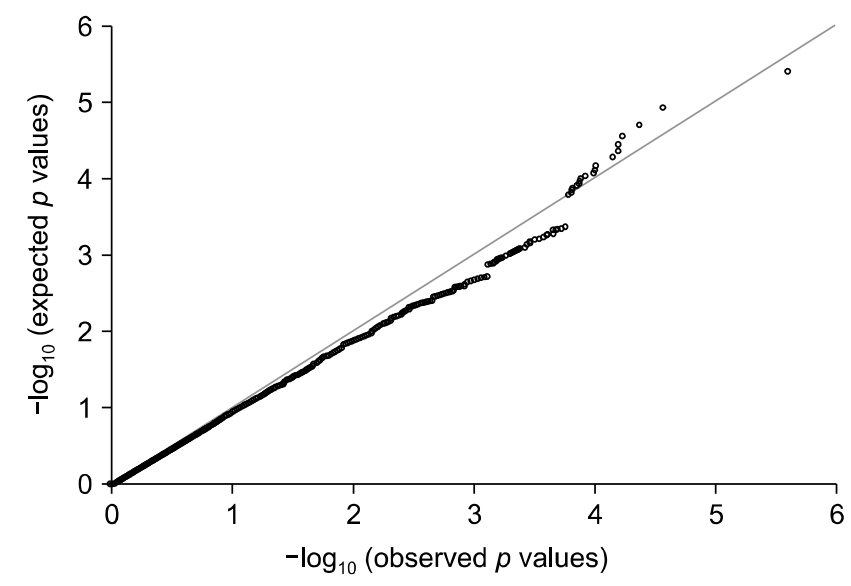

Fig. 2. The quantile-quantile (Q-Q) plot of 172,065 single nucleotide polymorphisms from 32 cases and 16 controls. The Q-Q plot graph from the continuous variable association with $\lambda=0.987$ demonstrating no population stratification as seen by the consistent deviation from the line (consistency in the observed $p$ values deviating from the expected $p$ values).
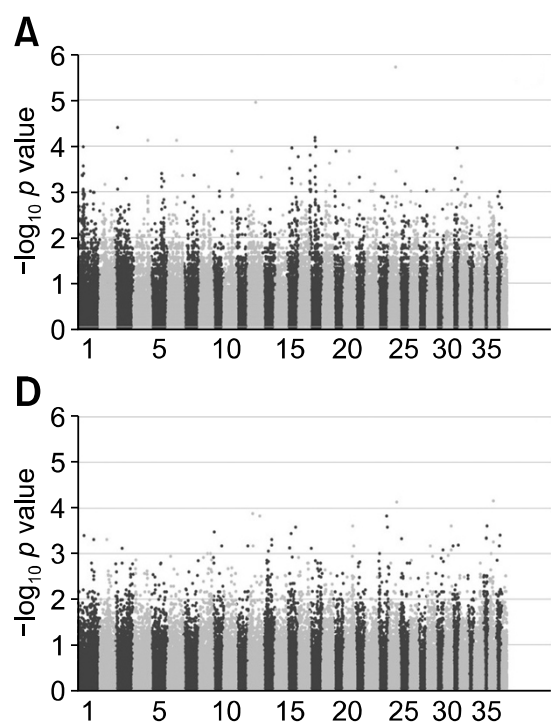
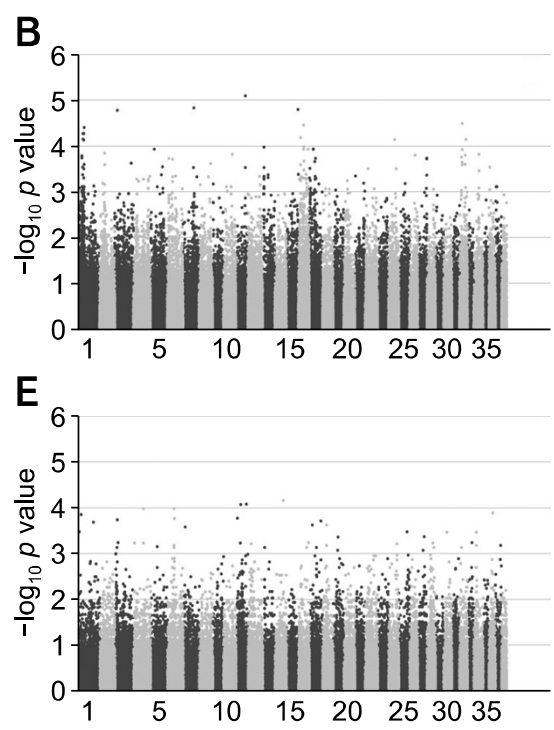
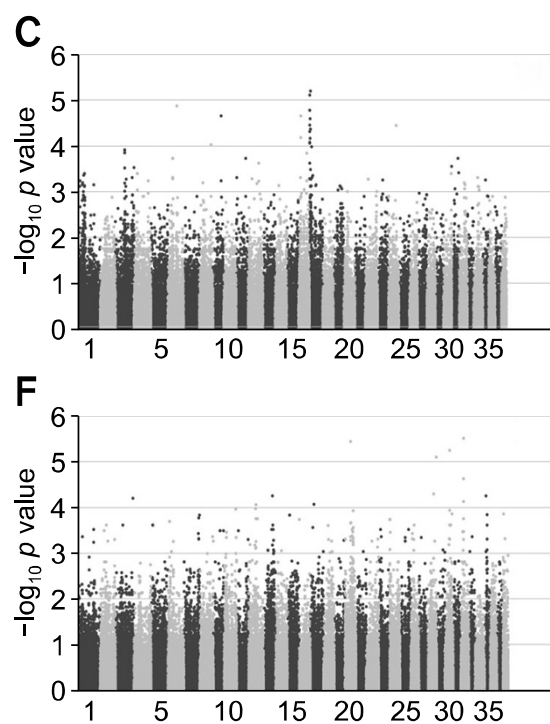

Fig. 3. Manhattan plots of the results of genome-wide association studies of each assessed condition. The conditions were degenerative mitral valve disease phenotype (A), American College of Veterinary Internal Medicine class (B), presence of mitral valve prolapse (C), leukocytosis (D), thrombocytosis (E), and vertebral heart scale group (F). The Fisher's exact test was applied to panels A, C, D and E in Fig. 3, and the linear regression test was applied to panels B and F in Fig. 3. Chromosome numbers are shown on the x-axis and results for each chromosome are shown in the diagram in black (odd-numbered chromosomes) and gray (even-numbered chromosomes). The $y$-axis is the $-\log _{10}$ of the calculated $p$ values. 


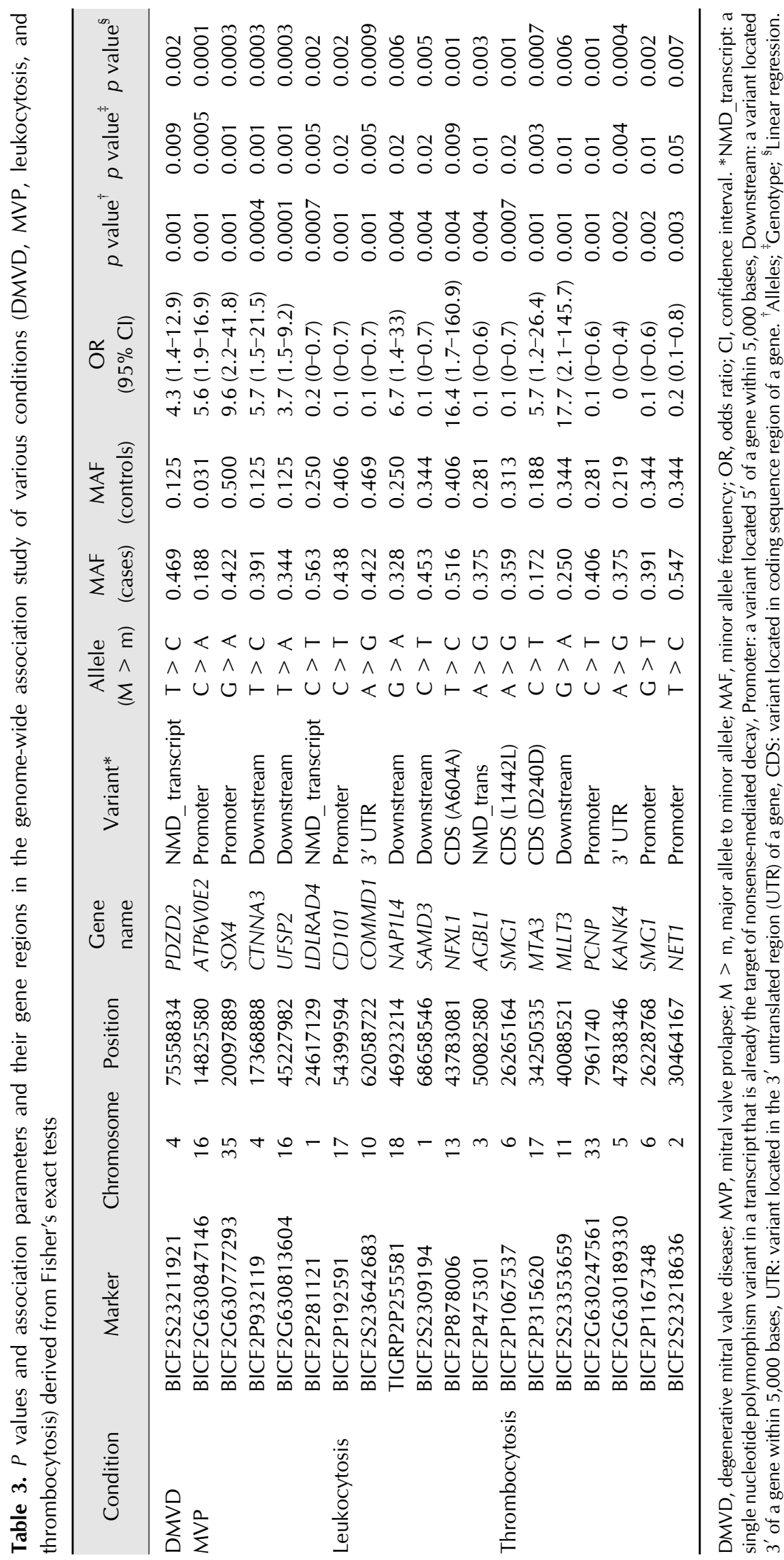


samples. Among these, 46,610 SNPs including those in the X chromosome were omitted, resulting in 127,052 SNPs to be used for further analysis. The overall genotype completion rate was $99.41 \%$. $P$ values of the Q-Q plot of the association test did not show obvious deviation $(\lambda$ value $=0.987)$ under the assumption of no genetic association, except in the right tail of the distribution (Fig. 2).

\section{Association analysis with candidate gene analysis of various conditions}

Manhattan plots were produced based on SNP-chip results using the allelic test to identify major loci associated with various conditions including DMVD phenotypes, ACVIM classes, presence of MVP, leukocytosis, thrombocytosis, and VHS. The $-\log _{10} p$ values were plotted against the physical map position of the SNPs (Fig. 3; chromosomes are alternatingly colored). Although the number of cases was insufficient for robust GWAS, the results should have detected major genes associated with a large proportion of the variance in the various conditions. However, there were few significant peaks of association that reached the 5\% threshold for genome-wide significance $\left(10^{-5}\right)$, as described in a previous study [11]. In the present study, the top $30 p$ value SNPs were sorted and their gene regions revealed (Supplementary Tables 1-6). The majority of SNPs for each condition were located in the intergenic region. However, several SNP variants were located in the region that can influence gene transcription. The SNPs with a high possibility to affect gene transcription are summarized in Tables 3 and 4. Table 3 shows the GWAS results of Fisher's exact tests for conditions consisting of two groups, while Table 4 shows GWAS results of linear regression tests for conditions that consist of greater than two groups.

\section{Discussion}

Artificial selection and tendencies within essentially independent breed populations could allow efficient detection of specific genetic associations with disorder traits that are present in specific breeds [27,35]. As described above, study of the genetic aspect of diseases in dogs may be a useful model for the assessment of human disorders. The multidimensional scaling plot in this study showed that the breed was sufficiently homogeneous with respect to the genetic background for a polygenic disorder, even within a limited number of cases and controls.

DMVD has been frequently reported in small-breed dogs weighing less than $9 \mathrm{~kg}$; however, body size is not likely to be related to the disease's onset and aggravation [30]. Because the genomes of small-breed dogs carry a variety of mutations that result in their size, they also may have linked genes that might elevate their susceptibility to DMVD [26]. Thus, the genetic transmission of DMVD could be revealed through precision

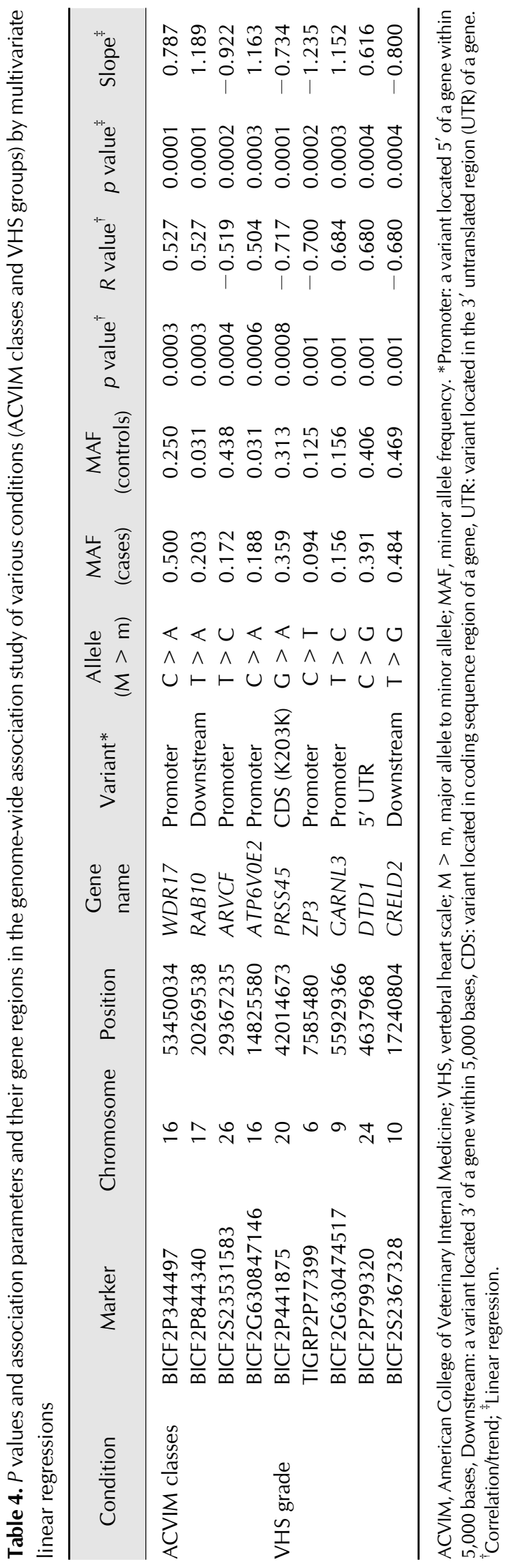

Journal of Veterinary Science 
mapping of concentrated markers in susceptible breeds. Recently, one study identified DMVD-associated loci in Whippets dogs, which is a large dog breed with a tendency to exhibit DMVD [31]. In the present study, Maltese dogs were evaluated because they are the most common breed that presents at animal hospitals in Korea. According to previous research (unpublished data), Maltese dogs are also the most represented breed $(n=60 / 170 ; 35.3 \%)$ among all cases of valvular heart disease. Therefore, Maltese dogs were considered the most suitable breed for genetic analysis of mitral valve disease in Korea.

Genetic linkage studies in human medicine have revealed loci associated with MVP on several chromosomes [10]. A recent GWAS of 1,412 MVP cases and 2,439 controls revealed LMCD1 (LIM and cysteine-rich domains 1), which encodes a transcription factor, to be significantly correlated with disease [23]. In spite of the results in these various studies, the causative gene or genes have not yet been identified. Several veterinary studies have been conducted to reveal genetic relationships associated with DMVD [2,35]. Studies have been performed to identify genes associated with mitral valve disease in CKCSs, with two loci identified in one study [20], but none in another [11]. These studies were performed in CKCS, in which DMVD occurs in nearly $100 \%$ of individuals. However, unlike previous research in CKCS, the present study was conducted in Maltese dogs, a breed without a 100\% incidence of DMVD. In addition, the present study included controls and cases diagnosed in old age (greater than 10 years), and the average age of the control group (13.5) was notably older than the DMVD group (10.41).

The association analysis in the present study used data for DMVD cases and controls. The most highly DMVD-associated genes were identified for five subject groups, including ACVIM class and presence of MVP, leukocytosis, and thrombocytosis, and VHS grade. Because the DMVD pathway is complex [2], evaluation of various factors is required. In the plot of SNPs associated with the presence of MVP, several significant SNPs were identified on chromosome 17. The observation that more SNPs were associated with MVP than with DMVD suggests the possibility of genetic progression of MVP.

Recent echocardiographic studies of MVP in dogs have described leaflet protrusion into the left atrium during systole $[29,33]$. The pathology of this disease in dogs may appear with primary and secondary effects. MVP in dogs is characterized by myxomatous valvular degeneration due to valvular thickening and mitral regurgitation [22]. However, recent studies have suggested that the pathology of MVP in dogs may be very similar to that of primary MVP in humans [9,24], with this possibly being a hereditary disease in dogs as it is in humans. As such, MVP in dogs could be considered a genetic disease, a possibility confirmed by the results of the present analyses.

The top 30 SNPs associated with each of the various conditions assessed in this study are shown in individual tables (Supplementary Tables 1-6). SNPs near intron sequences might affect RNA splicing and reduce or remove protein function, depending on the gene, and may cause disease. Additionally, introns may have their own transcription units that produce regulatory RNAs or small proteins; SNPs in these locations may affect the corresponding products of intron expression [36].

Several SNPs were located within sensitive transcript sequences, which could affect protein function and structure. One SNP was located in a transcript that was previously identified in a GWAS of the DMVD phenotype as the target of nonsense-mediated decay of the PDZD2 gene. This gene has been shown to interact with plakophilin 4 (PKP4), an Armadillo-like protein, characterized by a series of armadillo repeats, typically 42 to 45 amino acids in length. These proteins merge to the cytoplasmic portions of glycoproteins, resulting in molding plaques to which packages of cytoskeletal filaments adhere $[12,13]$. Genetic changes of plakoglobin protein in humans and mice were studied as result in cardiac disorders that could affect both adhesion and signaling problems [13]. This observation suggests the possible presence of signaling pathway problems, which supports one hypothesis regarding DMVD pathology.

The 30 most significant SNPs associated with ACVIM classes that affected the coding sequence were located in WD repeat domain 17 (WDR17), RAB10, ATPase, $\mathrm{H}+$ transporting V0 subunit e2 (ATP6VOE2), and ARVCF. ARVCF was shown to have a genetic mutation responsible for velo-cardio-facial syndrome, a common autosomal dominant human disorder with phenotypes including congenital cardiac defects [4].

Among the top 30 SNPs associated with MVP, CTNNA3 carries a protein within the vinculin/alpha-catenin family. This protein is responsible for cell-to-cell adhesion of muscular cells. Mutations of the gene are relevant to arrhythmogenic right ventricular cardiomyopathy [16], a congenital cardiomyopathy associated with remarkable cardiovascular morbidity and sudden death in the young [3]. This study revealed an association between MVP and a SNP located on a gene associated with congenital cardiac disease. This is not a matter of simply identifying DMVD; the finding also presents the potential for prolapse, which can be caused by other cardiac factors. Another SNP was located at LDLRAD4. This protein acts as a negative mediator of transforming growth factor beta (TGF- $\beta$ ) signaling and is possibly responsible for extracellular matrix production, motility, apoptosis, cell proliferation, differentiation, and immunosuppression [15]. Elevated TGF- $\beta$ expression has been observed in human and canine myxomatous valves. Their receptors are also up-regulated in DMVD [2]. As described above, it is possible that SNPs in genes regulated by TGF- $\beta$ are closely related to DMVD.

The most significant SNPs associated with leukocytosis included SAMD3, CD101, COMMD1, NAPIL4, NFXL1, and 
$A G B L 1 . S A M D 3$ is a protein-coding gene that is involved in the expression and phosphorylation of TGF- $\beta 1, S M A D 3$, and SAMD2, and cell cycle negative regulators were reported to decrease during the proliferative state but increase during the differentiation state [8]. As mentioned above, TGF- $\beta$ is involved in the pathogenic signaling in DMVD.

This study had several limitations. First, the small sample size made it difficult to determine if the differences in association values were caused by genomic variation and phenotype or a part of genomic variation resulting from the small number of samples. Second, while this study identified genetic variations, they were not verified at a protein level.

In conclusion, the present study evaluated the genetic relationship between SNPs significantly associated with DMVD and a variety of related conditions. DMVD was confirmed to be associated with these genetic variations, and the most prevalent genetic predisposition was the presence of MVP. To the best of our knowledge, this is the first report of a genetic predisposition to DMVD in Maltese dogs. The data could be the basis for further research on the genetic predisposition to MVP and DMVD in Maltese dogs.

\section{Acknowledgments}

Prof. Han at Chonbuk National University provided important advice during this research.

\section{Conflict of Interest}

The authors declare no conflicts of interest.

\section{References}

1. Atkins C, Bonagura J, Ettinger S, Fox P, Gordon S, Haggstrom J, Hamlin R, Keene B, Luis-Fuentes V, Stepien R. Guidelines for the diagnosis and treatment of canine chronic valvular heart disease. J Vet Intern Med 2009, 23, $1142-1150$

2. Aupperte H, Disatian S. Pathology, protein expression and signaling in myxomatous mitral valve degeneration: comparison of dogs and humans. J Vet Cardiol 2012, 14, 59-71.

3. Basso C, Fox PR, Meurs KM, Towbin JA, Spier AW, Calabrese F, Maron BJ, Thiene G. Arrhythmogenic right ventricular cardiomyopathy causing sudden cardiac death in boxer dogs: a new animal model of human disease. Circulation 2004, 109, 1180-1185.

4. Bonné S, van Hengel J, van Roy F. Chromosomal mapping of human armadillo genes belonging to the $120^{\mathrm{cth}} /$ plakophilin subfamily. Genomics 1998, 51, 452-454.

5. Borgarelli M, Savarino P, Crosara S, Santilli RA, Chiavegato D, Poggi M, Bellino C, La Rosa G, Zanatta R, Haggstrom J, Tarducci A. Survival characteristics and prognostic variables of dogs with mitral regurgitation attributable to myxomatous valve disease. J Vet Intern Med 2008, 22, 120-128.

6. Borgarelli M, Zini E, D'Agnolo G, Tarducci A, Santilli RA, Chiavegato D, Tursi M, Prunotto M, Häggström J. Comparison of primary mitral valve disease in German Shepherd dogs and in small breeds. J Vet Cardiol 2004, 6, 27-34.

7. Buchanan JW, Bücheler J. Vertebral scale system to measure canine heart size in radiographs. J Am Vet Med Assoc 1995, 206, 194-199.

8. Chen M, Lv Z, Huang L, Zhang W, Lin X, Shi J, Zhang W, Liang R, Jiang $\mathbf{S}$. Triptolide inhibits TGF- $\beta 1$-induced cell proliferation in rat airway smooth muscle cells by suppressing Smad signaling. Exp Cell Res 2015, 331, 362-368.

9. Delling FN, Vasan RS. Epidemiology and pathophysiology of mitral valve prolapse: new insights into disease progression, genetics, and molecular basis. Circulation 2014 , 129, 2158-2170.

10. Freed LA, Aciemo JS Jr, Dai D, Leyne M, Marshall JE, Nesta F, Levine RA, Slaugenhaupt SA. A locus for autosomal dominant mitral valve prolapse on chromosome 11p15.4. Am J Hum Genet 2003, 72, 1551-1559.

11. French AT, Ogden R, Eland C, Hemani G, Pong-Wong R, Corcoran B, Summers KM. Genome-wide analysis of mitral valve disease in Cavalier King Charles Spaniels. Vet J 2012, 193, 283-286.

12. Green KJ, Gaudry CA. Are desmosomes more than tethers for intermediate filaments? Nat Rev Mol Cell Biol 2000, 1, 208-216.

13. Grossmann KS, Grund C, Huelsken J, Behrend M, Erdmann B, Franke WW, Birchmeier W. Requirement of plakophilin 2 for heart morphogenesis and cardiac junction formation. $\mathrm{J}$ Cell Biol 2004, 167, 149-160.

14. Hinrichs AL, Larkin EK, Suarez BK. Population stratification and patterns of linkage disequilibrium. Genet Epidemiol 2009, 33 (Suppl 1), S88-92.

15. Hong KW, Shin MS, Ahn YB, Lee HJ, Kim HD. Genomewide association study on chronic periodontitis in Korean population: results from the Yangpyeong health cohort. J Clin Periodontol 2015, 42, 703-710.

16. Janssens B, Mohapatra B, Vatta M, Goossens S, Vanpoucke G, Kools P, Montoye T, van Hengel J, Bowles NE, van Roy F, Towbin JA. Assessment of the CTNNA3 gene encoding human $\alpha \mathrm{T}$-catenin regarding its involvement in dilated cardiomyopathy. Hum Genet 2003, 112, 227-236.

17. Karlsson EK, Lindblad-Toh K. Leader of the pack: gene mapping in dogs and other model organisms. Nat Rev Genet 2008, 9, 713-725.

18. Kraetschmer S, Ludwig K, Meneses F, Nolte I, Simon D. Vertebral heart scale in the beagle dog. J Small Anim Pract 2008, 49, 240-243.

19. Lindblad-Toh K, Wade CM, Mikkelsen TS, Kartsson EK, Jaffe DB, Kamal M, Clamp M, Chang JL, Kulbokas EJ 3rd, Zody MC, Mauceli E, Xie X, Breen M, Wayne RK, Ostrander EA, Ponting CP, Galibert F, Smith DR, DeJong PJ, Kirkness E, Alvarez P, Biagi T, Brockman W, Butler J, Chin CW, Cook A, Cuff J, Daly MJ, DeCaprio D, Gnerre S, Grabherr M, Kellis M, Kleber M, Bardeleben C, Goodstadt L, Heger A, Hitte C, Kim L, Koepfli KP, Parker HG, Pollinger JP, Searle SM, Sutter NB, Thomas R, Webber C, Baldwin J, Abebe A, Abouelleil A, Aftuck L, Ait-Zahra M, 
Aldredge T, Allen N, An P, Anderson S, Antoine C, Arachchi H, Aslam A, Ayotte L, Bachantsang P, Barry A, Bayul T, Benamara M, Berlin A, Bessette D, Blitshteyn B, Bloom T, Blye J, Boguslavskiy L, Bonnet C, Boukhgalter B, Brown A, Cahill P, Calixte N, Camarata J, Cheshatsang Y, Chu J, Citroen M, Collymore A, Cooke P, Dawoe T, Daza R, Decktor K, DeGray S, Dhargay N, Dooley K, Dooley K, Dorje $\mathbf{P}$, Dorjee $\mathrm{K}$, Donis $\mathrm{L}$, Duffey $\mathbf{N}$, Dupes A, Egbiremolen O, Elong R, Falk J, Farina A, Faro S, Ferguson D, Ferreira P, Fisher S, FitzGerald M, Foley K, Foley C, Franke A, Friedrich D, Gage D, Garber M, Gearin G, Giannoukos G, Goode T, Goyette A, Graham J, Grandbois E, Gyaltsen K, Hafez N, Hagopian D, Hagos B, Hall J, Healy C, Hegarty R, Honan T, Hom A, Houde N, Hughes L, Hunnicutt L, Husby M, Jester B, Jones C, Kamat A, Kanga B, Kells C, Khazanovich D, Kieu AC, Kisner P, Kumar M, Lance K, Landers T, Lara M, Lee W, Leger JP, Lennon N, Leuper L, LeVine S, Liu J, Liu X, Lokyitsang Y, Lokyitsang T, Lui A, Macdonald J, Major J, Marabella R, Maru K, Matthews C, McDonough S, Mehta T, Meldrim J, Melnikov A, Meneus L, Mihalev A, Mihova T, Miller K, Mittelman R, Mlenga V, Mulrain L, Munson G, Navidi A, Naylor J, Nguyen T, Nguyen N, Nguyen C, Nguyen T, Nicol R, Norbu N, Norbu C, Novod N, Nyima T, Olandt P, O'Neill B, O'Neill K, Osman S, Oyono L, Patti C, Penin D, Phunkhang P, Pierre F, Priest M, Rachupka A, Raghuraman S, Rameau R, Ray V, Raymond C, Rege F, Rise C, Rogers J, Rogov P, Sahalie J, Settipalli S, Sharpe T, Shea T, Sheehan M, Sherpa N, Shi J, Shih D, Sloan J, Smith C, Sparrow T, Stalker J, Stange-Thomann N, Stavropoulos S, Stone C, Stone S, Sykes S, Tchuinga P, Tenzing P, Tesfaye S, Thoulutsang D, Thoulutsang Y, Topham K, Topping I, Tsamla T, Vassiliev H, Venkataraman V, Vo A, Wangchuk T, Wangdi T, Weiand M, Wilkinson J, Wilson A, Yadav S, Yang S, Yang X, Young G, Yu Q, Zainoun J, Zembek L, Zimmer A, Lander ES. Genome sequence, comparative analysis and haplotype structure of the domestic dog. Nature 2005, 438, 803-819.

20. Madsen MB, Olsen LH, Häggström J, Höglund K, Ljungvall I, Falk T, Wess G, Stephenson H, Dukes-McEwan J, Chetboul V, Gouni V, Proschowsky HF, Cirera S, Karlskov-Mortensen P, Fredholm M. Identification of 2 loci associated with development of myxomatous mitral valve disease in Cavalier King Charles Spaniels. J Hered 2011, 102 (Suppl 1), S62-67.

21. Manolio TA. Genomewide association studies and assessment of the risk of disease. N Engl J Med 2010, 363, 166-176.

22. Nakayama T, Wakao Y, Nemoto H, Uechi M, Kageyama T, Muto M, Takahashi M. Mitral valve protrusion assessed by use of B-mode echocardiography in dogs with mitral regurgitation. Am J Vet Res 1996, 57, 791-797.
23. Nesta F, Leyne M, Yosefy C, Simpson C, Dai D, Marshall JE, Hung J, Slaugenhaupt SA, Levine RA. New locus for autosomal dominant mitral valve prolapse on chromosome 13: clinical insights from genetic studies. Circulation 2005, 112, 2022-2030.

24. Olsen LH, Fredholm M, Pedersen HD. Epidemiology and inheritance of mitral valve prolapse in Dachshunds. J Vet Intern Med 1999, 13, 448-456.

25. Ostrander EA, Wayne RK. The canine genome. Genome Res 2005, 15, 1706-1716.

26. Parker HG, Kilroy-Glynn P. Myxomatous mitral valve disease in dogs: does size matter? J Vet Cardiol 2012, 14, 19-29.

27. Parker HG, Kim LV, Sutter NB, Carison S, Lorentzen TD, Malek TB, Johnson GS, DeFrance HB, Ostrander EA, Kruglyak L. Genetic structure of the purebred domestic dog. Science 2004, 304, 1160-1164.

28. Pearson TA, Manolio TA. How to interpret a genome-wide association study. JAMA 2008, 299, 1335-1344.

29. Pedersen HD, Kristensen BO, Lorentzen KA, Koch J, Jensen AL, Flagstad A. Mitral valve prolapse in 3-year-old healthy Cavalier King Charles Spaniels. An echocardiographic study. Can J Vet Res 1995, 59, 294-298.

30. Raggi P, Callister TQ, Lippolis NJ, Russo DJ. Is mitral valve prolapse due to cardiac entrapment in the chest Cavity? A CT view. Chest 2000, 117, 636-642.

31. Stem JA, Hsue W, Song KH, Ontiveros ES, Luis Fuentes V, Stepien RL. Severity of mitral valve degeneration is associated with chromosome 15 loci in whippet dogs. PLoS One 2015, 10, e0141234.

32. Sutter NB, Eberle MA, Parker HG, Pullar BJ, Kirkness EF, Kruglyak L, Ostrander EA. Extensive and breed-specific linkage disequilibrium in Canis familiaris. Genome Res 2004, 14, 2388-2396.

33. Terzo E, Di Marcello M, McAllister H, Glazier B, Lo Coco D, Locatelli C, Palemo V, Brambilla PG. Echocardiographic assessment of $537 \mathrm{dogs}$ with mitral valve prolapse and leaflet involvement. Vet Radiol Ultrasound 2009, 50, 416-422.

34. Thomas WP, Gaber CE, Jacobs GJ, Kaplan PM, Lombard CW, Moise NS, Moses BL. Recommendations for standards in transthoracic two-dimensional echocardiography in the dog and cat. J Vet Intern Med 1993, 7, 247-252.

35. Tsai KL, Noorai RE, Star-Moss AN, Quignon P, Rinz CJ, Ostrander EA, Steiner JM, Murphy KE, Clark LA. Genome-wide association studies for multiple diseases of the German Shepherd Dog. Mamm Genome 2012, 23, 203-211.

36. Wang GS, Cooper TA. Splicing in disease: disruption of the splicing code and the decoding machinery. Nat Rev Genet $2007,8,749-761$. 